\title{
4D Visualization of Painted Sculpture and Murals
}

\author{
Ai MingYao ${ }^{1,2}$, Tong $\mathrm{Hua}^{3}$, Shen $\mathrm{Li}^{1}$, Wang RuoXi ${ }^{1,2, *}$, Zhang Fang ${ }^{2}$, Zhang ZhiChao ${ }^{1}$, \\ Hu QingWu ${ }^{1}$, Zhu YiXuan ${ }^{1,2}$, Zhang Heng ${ }^{3}$ \\ ${ }^{1}$ School of Remote Sensing and Information Engineering, \\ ${ }^{2}$ State Key Laboratory of Information Engineering in Surveying, Mapping and Remote Sensing, \\ ${ }^{3}$ College of Chemistry and Molecular Sciences, \\ Wuhan University, Wuhan 430079, China \\ rxwang@whu.edu.cn
}

KEY WORDS: Chemical Analysis, Image Segmentation, 3D Reconstruction, 4D Visualization, Painted Sculpture and Murals

\begin{abstract}
:
Most cultural heritage applications address visualization with using various media or platforms: desktop-based multimedia presentations, museum kiosks, or videos produced with computer animation. However, these techniques can not directly reveal or show the course that the colorful surface of painted sculpture and murals becomes faint along with the change of the climate and time. Most current techniques just preserve the current appearance and disseminate the current situation of the painted sculpture and murals. The course how these forms of cultural heritage change along the time has not been visualized. In this paper we developed an approach to modelling of painted sculpture and murals that has undergone changes over the years. Different hypotheses has also be given if there is uncertainty. A painted sculpture of Mogao Grottoes is used to demonstate this approach.
\end{abstract}

\section{INTRODUCTION}

Painted sculpture and murals are two common important forms of culture heritage existing in many drought-prone water-scarce areas, such as Mogao Grottoes at Dunhuang in western China, Afghanistan, and the Middle East. Sculpture and paintings are not simply painted layer by layer of pigment, while they are creations full of spirits owning to ancient painters aura, the pursuit of beauty in life, and their souls' vents as well. But the pigment is the direct material manifestation of painting art(Ma, Guo, et al., 2003). However, the long-time natural efflorescence and disasters, such as earthquakes and floods, can cause huge damage on the uncovered painted sculpture and murals, which leads to flaking, hollowing, shedding, fading, and discolouration. The increasing tourists make this damage much worse. The painted sculpture and murals of Mogao Grottoes at Dunhuang suffer from these damages heavily. These diseases are resulted from the complex chemical and physical interactions over hundreds of years of from the view of mechanism theory. Without considering the physical surface changing, namely flaking, hollowing and shedding, the mechanism of colour changing can be used to restore the original appearances of the murals and painted sculptures.

The mechanism of colour changing does not only offer a visual medium for the interactions among those cultural relics protectors, but also defines the rules that can be used to predict the trends of changes in the external factors. This can contribute to effective preservations and maintenance in case. In addition, the simulation of colour changing can also help people understand and appreciate the evolution processes of the murals in a visual-friendly mode. This model is closer to the original creations in natural conditions from the view of arts(Pan and $\mathrm{Lu}$, 2003).

Evolution of colour actually is the result of complex physical and chemical reaction of pigment. A large number of studies have shown that the pigments of Dunhuang murals and painted sculptures are mostly inorganic mineral pigments, and in most cases they are made from natural mineral, such as vermilion and vandyke red used as red pigments, lapis lazuli and azurite used as blue pigments, verde antico and copper chloride (including natural patina) used as green pigments, aolinite, calcite and gypsum used in white paint, and iron oxide black used as black pigments. Natural mineral pigments, in normal conditions are stable. Therefore, the red colour painted with vandyke red and vermilion, blue colour with lapis lazuli and azurite, green colour with verde antico and copper chloride, and white with aolinite, calcite and gypsum in the Mogao Grottoes in Dunhuang frescoes keep little discolouration after hundreds of years of vicissitudes. Vermilion has a different degree of dimming, and the colour and brightness of vermilion grows less gorgeous than the original, while the hue does not change. Ultramarine, an artificial mineral pigment, is relatively stable as well, but only the man-made inorganic pigments of minium shows severe discolouration( $\mathrm{Li}, 2002)$.

Motivated by the change of colour caused by chemical reaction of minium and the interest in sharing the gorgeous original appearance, this paper analyses the collected the minium pigment sample in Mogao Grottoes, and experiment on the chemical mechanism of discolouration of minium to simulate the process of discolouration of minium in the case of controlled temperature and humidity. Then the change of red is showing via a texture 3D model of a statue of Ananda Sagara statue in 159 cave of Dunhuang Mogao Grottoes. This approach achieves the $4 \mathrm{D}$ visualization of sculptures and murals.

\section{RELEATED WORK}

A lot of research works have been proposed in the literature for digitalized protection and visualization of cultural heritage. While laser scanning, photogrammetry and computer vision approaches have been used extensively to record, measure, and preserve cultural heritage sites, they are only capable of displaying the current state of the environment with 3D model(Laycock, Drinkwater, et al., 2008). Though the 
interaction and navigation within virtual 4D worlds(adding time to $3 \mathrm{D}$ worlds) may be problematical due to the time dimension(El-Hakim, MacDonald, et al., 2006), a method like dealing with $4 \mathrm{D}$ processing (3D objects plus the time) is not a novel and unique concept as the study of urban transformations has become an important axis in the investigation of the city with long history and culture(PATIAS, KAIMARIS, et al., 2011). The key to obtain $4 \mathrm{D}$ information or $4 \mathrm{D}$ reconstruction is to get the proper data over time, mainly old data. There are several approaches demonstrate their generation of 4D (geometry + time) model of the cultural heritage cites or artifacts. Old photos are not used as the only main data source for geometric reconstruction through time(Doulamis, Ioannides, et al., 2013, El-Hakim, MacDonald, Lapointe, Gonzo and Jemtrud, 2006, Hadjiprocopis, Ioannides, et al., 2014), but are used for texture mapping(Guidi and Russo, 2011, Laycock, Drinkwater and Day, 2008). Old maps and even an oldwooden model can be used as a starting point of 4D reconstruction(Kersten, Keller, et al., 2012, Laycock, Drinkwater and Day, 2008). Historical aerial photographs are also used with dense image matching algorithms to realize 3d models of a city in different years(Adami, 2015). Other researchers lay the simulation of colour changing and fading process of ancient Chinese wall paintings on chemical-based modeling for 4D reconstruction. Chemical mechanism of colour changing mainly caused of oxidization is modeling the colour change for aging simulation(Shi and $\mathrm{Lu}, 2005$ ). In addition, the empirical knowledge on contents and styles from artists and experts can help complete the 4D model(El-Hakim, MacDonald, Lapointe, Gonzo and Jemtrud, 2006, Shi, Lu, et al., 2006). In this paper, a novel approach is proposed on $4 \mathrm{D}$ visualization of the process of color change based on the changing mechanism of the pigments, esperially minum(Guo, Duan, Wang, et al., 1995, Li, 1992, Li, Fan, et al., 1992).

\section{METHODOLOGY}

\subsection{Chemical Analysis on Colour Changing of Minum}

Based on the principles of colourimetry and painting art, the colour of a mixture is essentially as a result of the combination of different ingredients. Therefore, the key step for colour changing simulation is to obtain the relationship between colour and the percentages of different pigment components. In other words, if the colour and the pigment components could be easily acquired, then the chemical ingredients could be identified as well as the correspondence relationship between material and colour is obtained. In this paper, we focused on the change of red colour caused by minium with lead tetraoxide $\left(\mathrm{Pb}_{3} \mathrm{O}_{4}\right)$ as its main ingredient.

It is already known that the red colour of minum will change into black colour under the complex chemical and physical interactions over hundreds of years( $\mathrm{Li}, 1992)$. Therefore, the next work to be done is to explore the rate of chemical changing of minum, and to measure the dynamic colour over time. Then we designed an experiment to investigate the chemical kinetics rules of the dynamic changes of colour and the pigment materials in the case of the oxidization process from red minum $\left(\mathrm{Pb}_{3} \mathrm{O}_{4}\right)$ to black lead dioxide $\left(\mathrm{PbO}_{2}\right)$. The pigment changes clearly demonstrated that the colour of minum changes from red to black. Meantime, we conducted the integration computation on the whole optical spectrum and obtained the colour in Lab values of the colour-changing minum. Based on calculated colour values, we built the corresponding relationship between the pigment material attribute variables and its colour values to retrieval and represent the pigment colon usage of the mural paintings.

The pigment colour retrieval is actually the inverse process of colours gradually changing over time. The colour condition demonstrated at certain specific time is in fact the colour representation at that time period. The bridge to link colours to time is the chemical components of pigment itself. In the example of minum, we can obtain the $\mathrm{Pb}$ contents of variant valences for different time periods based on modern equipment and detecting methods. This can help construct the functional relationship between the colour values and their corresponding times, which quantitatively relates time and colours to realize colour retrieval and representation.

The aforementioned experiment and analysis also shows the intermediate variable between time and colour - the chemical component which also relates the time and colour in equations. The time equation and colour equations are respectively shown in Equation $\mathbf{1}$ and 2:

$$
\begin{aligned}
& X=F(t) \\
& \mathrm{C}_{\mathrm{L}}=L(X) \\
& \mathrm{C}_{\mathrm{a}}=A(X) \\
& \mathrm{C}_{\mathrm{b}}=B(X)
\end{aligned}
$$

The time $\mathrm{t}$ in Equation $\mathbf{1}$ is the aging time in experimental conditions, $X$ is the corresponding content percentage of $\mathrm{Pb}$. The $\mathrm{C}_{\mathrm{L}}, \mathrm{C}_{\mathrm{a}}, \mathrm{C}_{\mathrm{b}}$ in Equation 2 is the colour under Lab colour system that changing minum shows. So we still need to convert it to the aging time in natural conditions proportionally. The red of minum is considered to be original colour of the painted sculptures and murals, and the black is suggested to be the current colour. This improvement of the colour changing is the colour aging model in this paper. This improvement can better help simulate the minum colour changing of Mogao Grottoes in natural conditions to deepen the understanding and matching of colour changing over time for those murals.

\subsection{Colourful 3D Modelling of Painted Sculpture}

High resolution handy 3D Laser Scanner is used to capture point cloud on the geometric surface of the painted sculpture. The point cloud of high density is processed into 3D triangulated mesh. But this is far from the need of view and sight. So the 3D mesh should be textured with high resolution images, which are taken with Canon EOS-1Ds Mark III and standard light curtain in order to ensure uniform light of texture images without spot. Generally, four flashing lights can basically meet the needs of the texture image data collection work for cultural heritage. Then, it is necessary to take the step of texture mapping. High precision texture mapping is still a difficult problem in high fidelity reconstruction.

For the sake of avoiding the effect of colour difference resulting from light, camera settings and environment and making the texture look like more authentic, an automatic image dodging technique based on automatic electric printer principle is adopted to obtain the consistency input for image texture mapping. The image dodging algorithm is designed to simulate the process of automatic electric printer by image process(Ai, Zhang, et al.,2014, Li and $\mathrm{Hu}, 2010)$. 
A non-rigid texture mapping method(Zhang, Huang, et al.), which combine the rigid perspective projection model and nonrigid adjustment processing together, is used to generate precise mapped texture. We adjust the mapping results at rich texture area, using the gradient constraint at edges in image to give parameters with weighted thin-plate spline. These two operation maps 6 uniform-colour texture images precisely onto the 3D geometric model either in the global or in the local areas.

\subsection{Texture Image Segmentation}

In this paper, two image segmentation algorithms, namely GrabCut and Watershed. Figure 1 shows the image segmentation processed by the two algorithms.

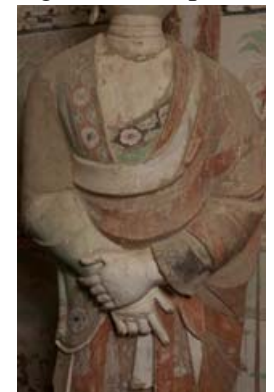

(a) Original Image

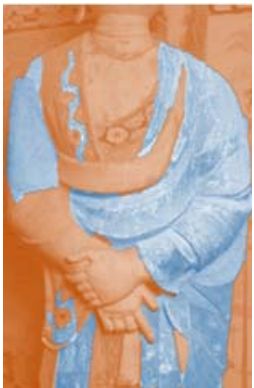

(b)GrabCut

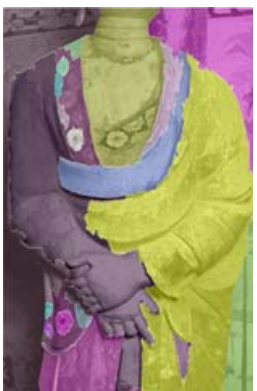

(c)Watershed
Figure 1. Texture image segmentations is acquired with GrabCut and Watershed algorithms.

The advantages of GrabCut algorithm over Watershed algorithm are that priori expert knowledge could be added, and that several amendments on foreground and background image regions could be processed to achieve more reasonable results of segmentation. GrabCut gets good segmentation in the local region decorated with detail clothing patterns. This algorithm also separates the flower pattern from the red background with some human intervention, which is beneficial to obtain the precise classes of texture image and better colour inversion. The advantage of Watershed algorithm is that it is sensitive to the red colour with varying degrees of discolouration. Since the red colour is different on the surface of Ananda Sagara statue in 159 cave of Dunhuang Mogao Grottoes, the segmentation of Watershed algorithm in red regions is reasonable. However, it is inconvenient that the unconnected regions with similar discolouration need to be manually marked, merged and extracted. Besides that, it is difficult for the Watershed algorithm to distinguish the blue and green flower patterns from the red regions. Therefore, Watershed algorithm applied to the segmentation of single colour regions with little detailed patterns.

The comparison and analyst on the results of segmentation obtained by GrabCut and Watershed algorithms demonstrates that GrabCut succeeds in more precise segmentation due to several human-computer interactions. GrabCut could not only distinguishes finely different colour regions on the same texture image, but could extract the unconnected regions of similar colour with different degrees of discolouration. The six texture images are segmented by using GrabCut algorithms in the same way to generate the temporal texture of $3 \mathrm{D}$ geometric model with the colour aging model.

\subsection{Temporal Texture Generation and 4D Visualization}

In the real environment, the rate of discolouration and colour aging of only one kind of pigment would be different because of the different light, temperature and humidity. Thus the influence of the environment should be taken consideration into the colour aging model. We use weight to measure the impact of environment. Given the variable time ( $t$ in Equation 1), weights are set up according to the image regions of red discolouration. Then the recognition of varying degrees of red discolouration is the following operation to simulate the environmental effect. In order to avoid the joint pixels and overlap caused by further segmentation, supervised classification is adopted to sort the red image regions into three categories which represent the degrees of environmental impact. The classification is performed by the differences between the visual effects of these red regions. Then, the weights are signed to regions of three classes. Figure 2 displays the inversion of discolouration improved with the weight colour aging model. In Figure 2, large effect is put to the dark red regions, while the light red regions get less effect. Figure 2 also shows that the results of reclassification are close to the discolouration in the term of visual effect.

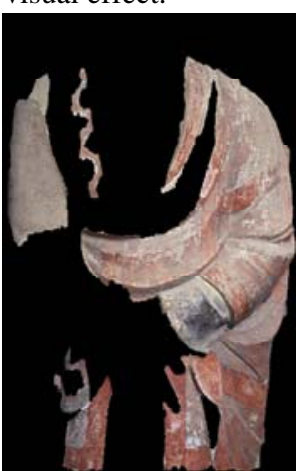

(a) Original Image

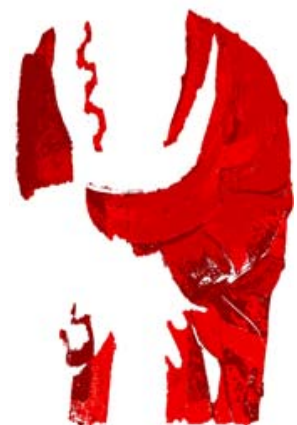

(b) Classified Image
Figure 2. Classification in red regions.

$4 \mathrm{D}$ visualization implemented in OpenGL simulates the process of colour aging with the colourful 3D model of Ananda Sagara statue. A time-axis is used to set up the time for visualization. The temporal texture images are real-time calculated according to the colour aging model described in section 3.1 in pace with the time changing, which makes sure that the visualized scene of the painted sculpture is in accordance with the inversion of colour. Users can observed the change of colour in different regions of the $3 \mathrm{D}$ texture model as the time goes.

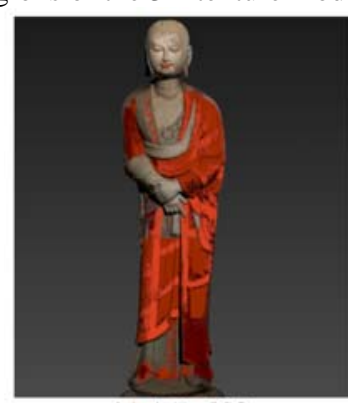

(a) A.D. 830

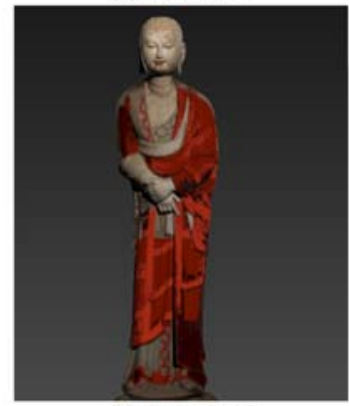

(c) A.D. 1700

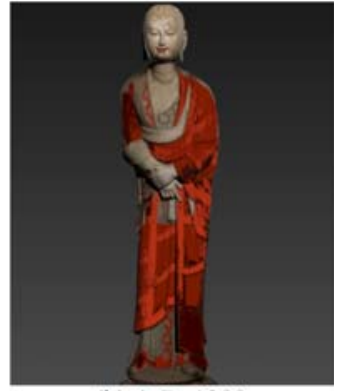

(b) A.D. 1200

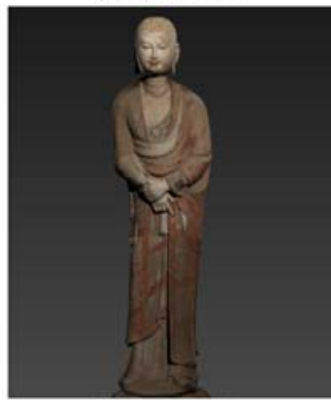

(d) A.D. 2013 
Figure 3. The texture images of 3D model is changing as the time flies.

Figure 3 shows the colour of the texture images on the 3D model changes along with the change of time. Figure 3(a) displays the original colour of the 3D model when the painted sculpture is created and designed in about A.D. 830, when the Tang Dynasty had been governed its people for half its lifespan. Figure 3(b) is the pictures in about A.D.1200. Figure 3(c) is the 3D colour-restored model as the time stops at A.D. 1700. Figure 3(d) shows the colourful 3D model at the current time when these images are taken in the year of 2013. When compared these figures, it is obvious that the change of colour is much faster in the early periods than in later times under the circumstances of the weighted colour aging model. They also illuminate that the aging reaction becomes slow when the time goes.

\subsection{Critical Analysis}

In fact, there are several issues that could be deeply discussed after this research. Each struggle greatly affected the process of how the research is critiqued and improved. The following text is a critical analysis of how to improve the research.

In this paper, the chemical components of the pigment are acquired by the chemical analysts in laboratory. It is the research origin of this paper that the main component of the black samples is minum. Our research is performed along with the discolouration of this pigment. There may be a doubt that minum is not the main pigment of red colour used in all the painted sculptures and murals. What is more, the colour is stable when minum is mixed with other pigments $(\mathrm{Li}, 2002$, $\mathrm{Su}$, $\mathrm{Hu}$, et al., 1996). Therefore, the method proposed in this paper can not be directly applied to other red pigments or mixture of pigments. There is no doubt that the method of this paper can be adopted to uses chemical analysis to gain the knowledge of the components of pigments, and then to get the original colour of the pigments, in which way the process of discolouration can be obtained and the 4D visualization can be achieved.

Meanwhile, 4D visualization proposed in this paper is based on the assumption that the colour of minum pigment changes equably over time, even though we divided the red regions into three classes of different discolouration. This assumption leads to the exact proportion of visualization condition and laboratory condition. However, the rate of discolouration of even one kind of pigment is inconsistent with the different conditions of light, temperature and humidity under real natural circumstance. In the perspective of whole statue, the influence of environment on the current colour of pigment is different in parts: large effect shows up in the dark-colour regions on the painted surface, while little effect in bright-colour regions. Given the impacts of environment, proper proportion of weights is signed to the large, medium and small levels of discolouration. Thus this way, at least from our point of view, is estimation for better visualization, which introduces subjectivity, which could not be verified. Be that as it may, this paper explores a path leading to the ancient painted sculptures and murals of original colours with chemical analysis, and gives the idea and examples of exhibiting the way.

\section{CONCLUSION AND FUTURE WORK}

To maintain of painted sculptures and murals as they really are is so great challenge that several generations have contribute their wholes to it. This paper tries to reveal the veil that the discolouration of the pigments puts on this form of cultural heritage. The chemical analysis methods are used to search the cause of discolouration and the very process of colour changing The aging experiment on the pigment of minum demonstrates that chemical element components of pigments become the bridge between the material and colour. We design the workflow to show the original colour of a dark-colour painted sculpture in Mogao Grottoes in Dunhuang. This workflow includes 3D modelling of painted sculpture, image segmentation of texture images, temporal texture generation and $4 \mathrm{D}$ visualization.

This paper will show the ordinary tourists the visual original red colour of the painted sculptures and murals. The 4D visualization of other colour or other pigments, for instance green and copper chloride, would be the future work. The real discolouration of pigments would be a challenge for long time. The visual effect would be more realistic if the texture image segmentation was performed more precisely and the weight of proportion was more reasonable. These works would be explored in the future to contribute better 4D visualization.

\section{ACKNOWLEDGEMENTS}

The authors would like to express their gratitude to the editors and the reviewers for their constructive and helpful comments for substantial improvement of this paper. This work is supported by National Basic Research Program of China (973 Program, No. 2012CB725300), and National Natural Science Foundation of China (No. 41271452).

\section{REFERENCES}

[1] A. Adami, "4D City transformations by time series of aerial images," International Archives of the Photogrammetry, Remote Sensing and Spatial Information Sciences - ISPRS Archives. pp. 339-344.

[2] M. Ai, Z. Zhang, F. Zhang, Q. Hu,Y. Zhu, "Tourists archive the 3D documentation of cultural heritage," Proceedings 2014 22nd International Conference on Geoinformatics, Geoinformatics 2014. pp. Aerial Survey Office, Forestry Bureau; National Land Surveying and Mapping Center.

[3] A. Doulamis, M. Ioannides, N. Doulamis, A. Hadjiprocopis, D. Fritsch, O. Balet, M. Julien, E. Protopapadakis, K. Makantasis, G. Weinlinger, P. S.Johnsons, M. Klein, D. Fellner, A. Stork,P. Santos, "4D reconstruction of the past," Proceedings of SPIE - The International Society for Optical Engineering. pp. European Space Agency (ESA); Intergraph (SG and I) Italia LLC, part of Hexagon; Geosystems Hellas; Frederick University; Neapolis University.

[4] S. F. El-Hakim, G. MacDonald, J.-F. Lapointe, L. Gonzo,M. Jemtrud, "On the digital reconstruction and interactive presentation of heritage sites through time," VAST: International Symposium on Virtual Reality, Archaeology and Intelligent Cultural Heritage. pp. 243-250.

[5] G. Guidi,M. Russo, "Diachronic 3D reconstruction for lost Cultural Heritage," International Archives of the Photogrammetry, Remote Sensing and Spatial Information Sciences - ISPRS Archives. pp. 371-376.

[6] A. Hadjiprocopis, M. Ioannides, K. Wenzel, M. Rothermel, P. S. Johnsons, D. Fritsch, A. Doulamis, E. Protopapadakis, G. Kyriakaki, K. Makantasis, G. Weinlinger, M. Klein, D. Fellner, A. Stork,P. Santos, "4D reconstruction of the past: The image retrieval and 3D model construction pipeline," Proceedings of SPIE - The International Society for Optical 
Engineering. pp. et al.; Euro-agriwot; European Cooperation in Science and Technology (COST); Geosystems Hellas; Intergraph; Li-Cor.

[7] T. P. Kersten, F. Keller, J. Saenger,J. Schiewe, "Automated generation of an historic 4D city model of hamburg and its visualisation with the GE engine," Lecture Notes in Computer Science (including subseries Lecture Notes in Artificial Intelligence and Lecture Notes in Bioinformatics). pp. 55-65.

[8] R. G. Laycock, D. Drinkwater,A. M. Day, "Exploring cultural heritage sites through space and time," Journal of Computing and Cultural Heritage, vol. 1, no. 2, 2008.

[9] P. PATIAS, D. KAIMARIS,E. STYLIANIDIS, "CHANGE DETECTION IN HISTORICAL CITY CENTERS USING MULTI-SOURCE DATA: THE CASE OF HISTORICAL CENTER OF NICOSIA - CYPRUS."

[10] X. Shi,D. Lu, "Colorimetric and chemical modeling based aging simulation of dunhuang murals," Proceedings - Fifth International Conference on Computer and Information Technology, CIT 2005. pp. 570-574.

[11] X. Shi, D. Lu,J. Liu, "Color Changing and Fading Simulation for Frescoes Based on Empirical Knowledge from Artists," Advances in Multimedia Information Processing PCM 2006, Lecture Notes in Computer Science Y. Zhuang, S.-Q. Yang, Y. Rui et al., eds., pp. 861-869: Springer Berlin Heidelberg, 2006.

[12] F. Zhang, X. Huang, W. Fang, Z. Zhang, D. Li,Y. Zhu, "Texture reconstruction of 3D sculpture using non-rigid transformation,” Journal of Cultural Heritage, no. 0.

[13] Guo Hong, Duan Xiuye, Wang Junhu, Li Jun, “Color stability of pigments of Mogao Grottoes at Dunhuang and its related problems," DUNHUANG RESEARCH, no. 03, pp. 47-58, 1995. (In Chinese)

[14] Li Qingquan, Hu Qingwu, “A Pavement Crack Image Analysis Approach Based on Automatic Image Dodging,” Journal of Highway and Transportation Research and Development, no. 04, pp. 1-5+27, 2010. (In Chinese)

[15] Li Zuixiong, "Research on Mogao Caves murals of red pigment and mechanism of its discolouration, " DUNHUANG RESEARCH, no. 03, pp. 41-54+128-129+135137, 1992. (In Chinese)

[16] Li Zuixiong, "Pigment Analysis On Tang Dynasty Murals at The Mogao Grottes,” DUNHUANG RESEARCH, no. 04, pp. 11-18+110, 2002. (In Chinese)

[17] Li Zuixiong, Fan Zaixuan, Sheng Fenling, “ New development of research on the colour changing of minum, cinnabar and vandyke red," DUNHUANG RESEARCH, no. 01, pp. 89-117+123-124+85, 1992. (In Chinese)

[18] Ma Zanfeng, Guo Hong, Wang Huizhen, Li Zuixiong, M. Schilling, "Color measurement research of Cave 85 at the Mogao Grottoes in Dunhuang, ” SCIENCES OF CONSERVATION AND ARCHAEOLOGY, no. 02, pp. 33-36, 2003. (In Chinese)

[19] Pan Yunhe, Lu Dongming, “Digital Protection and Restoration of Dunhuang Mural,” JOURNAL OF SYSTEM SIMULATION, no. 03, pp. 310-314, 2003. (In Chinese)

[20] Su Bomin, Hu Zhide, Li Zuixiong, "A Study of the Mixed Pigments Used in the Wall Paintings of Dunhuang,” DUNHUANG RESEARCH, no. 3, pp. 149-162, 1996. (In Chinese) 